\title{
SMALL FRACTIONAL PARTS OF POLYNOMIALS
}

\author{
ROGER BAKER
}

\begin{abstract}
Let $k \geq 6$. Using the recent result of Bourgain, Demeter, and Guth [5] on the Vinogradov mean value, we obtain new bounds for small fracitonal parts of polynomials $\alpha_{k} n^{k}+\cdots+\alpha_{1} n$ and additive forms $\beta_{1} n_{1}^{k}+\cdots+\beta_{s} n_{s}^{k}$. Our results improve earlier theorems of Danicic (1957), Cook (1972), Baker (1982, 2000), Vaughan and Wooley (2000), and Wooley (2013).
\end{abstract}

\section{INTRODUCTION}

Let $J_{s, k}(N)$ be the Vinogradov mean value,

$$
J_{s, k}(N):=\int_{[0,1)^{k}}\left|\sum_{n=1}^{N} e\left(x_{k} n^{k}+\cdots+x_{1} n\right)\right|^{2 s} d x_{1} \ldots d x_{k} .
$$

Here $s$ and $k$ are natural numbers. Recently Wooley [12] (for $k=3$ ) and Bourgain, Demeter, and Guth [5] (for $k \geq 4$ ) have established the main conjecture for $J_{s, k}(N)$, namely

$$
J_{s, k}(N) \ll_{k, \varepsilon} N^{s+\varepsilon}+N^{2 s-k(k+1) / 2+\varepsilon} .
$$

Here $\varepsilon$ is an arbitrary positive number. In the present note we combine (1.1) with techniques from two earlier publications [3,4] to obtain new bounds of the form

$$
\min _{1 \leq n \leq N}\left\|\alpha_{k} n^{k}+\cdots+\alpha_{1} n\right\| \ll_{k, \varepsilon} N^{-\mu_{k}+\varepsilon} \quad(k=8,9, \ldots)
$$

(with arbitrary real numbers $\alpha_{1}, \ldots, \alpha_{k}, \beta_{1}, \ldots, \beta_{s}$ here and below);

$$
\min _{1 \leq n \leq N}\left\|\alpha_{k} n^{k}+\alpha_{1} n\right\| \ll_{k, \varepsilon} N^{-\rho_{k}+\varepsilon} \quad(k=6,7, \ldots)
$$

$$
\min _{\substack{0 \leq n_{1}, \ldots, n_{s} \leq N \\\left(n_{1}, \ldots, n_{s}\right) \neq \mathbf{0}}}\left\|\beta_{1} n_{1}^{k}+\cdots+\beta_{s} n_{s}^{k}\right\| \ll N^{-\sigma_{s, k}+\varepsilon} \quad(k=6,7, \ldots, s \geq 1) .
$$

Theorem 1. Let $k \geq 8$. Then (i) holds with $\mu_{k}=1 / 2 k(k-1)$.

2010 Mathematics Subject Classification. Primary 11J54. 
Theorem 2. (a) Let $k \geq 6$. Then (ii) holds with $\rho_{k}=1 / k(k-1)$.

(b) Let $k \geq 6$. For a certain positive absolute constant B, (ii) holds with $\rho_{k}=1 / k(2 \log k+B \log \log k)$.

Theorem 3. (a) Let $k \geq 6,1 \leq s \leq k(k-1)$. Then (iii) holds with $\sigma_{s, k}=s / k(k-1)$.

(b) Let

$F(J, s, k)=\min \left(\frac{s}{J}, \max _{J+1 \leq h \leq s} \min \left(\frac{(2 h-2)(s-k)+4 k-4}{h(s-k)+4 h-4}, \frac{s-h+J+1}{J}\right)\right)$

Then (iii) holds for $k \geq 6, s>k(k-1)$ with

$$
\sigma_{s, k}=F(k(k-1), s, k) \text {. }
$$

In particular,

$$
\min _{\substack{0 \leq n_{1}, \ldots, n_{s} \leq N \\\left(n_{1}, \ldots, n_{s}\right) \neq \mathbf{0}}}\left\|\beta_{1} n_{1}^{6}+\cdots+\beta_{s} n_{s}^{6}\right\| \ll N^{-s / 30+\varepsilon}(1 \leq s \leq 56) .
$$

We note here the existing results in each case. Let $K=2^{k-1}$.

(i) This is known with $\mu_{k}=1 / K(2 \leq k \leq 8)$ (Baker [1]) and $\mu_{k}=1 / 4 k(k-2)$ for $k \geq 9$ (Wooley [11]).

(ii) Only the special case $\alpha_{1}=0$ has been considered separately from (i). Here the result is known with $\rho_{2}=4 / 7$ (Zaharescu [14]); $\rho_{k}=1 / \mathrm{K}$ $(3 \leq k \leq 6)$ (Danicic [7]), while there are the values $\rho_{7}=1 / 57.23$, $\rho_{8}=1 / 69.66, \rho_{9}=1 / 82.08, \rho_{10}=1 / 94.62, \rho_{11}=1 / 107.27, \ldots, \rho_{20}=$ $1 / 222.16$, given by Vaughan and Wooley [9], which are better than the present method gives (in the monomial case) for $k \geq 11$. There is an absolute positive constant $C$ such that, for $k \geq 6$,

$$
\min _{1 \leq n \leq N}\left\|\alpha n^{k}\right\| \ll_{k, \varepsilon} N^{-1 / k(\log k+C \log \log k)}
$$

(Wooley [10]).

(iii) This is known with $\sigma_{s, k}=s / K$ for $k \geq 2,1 \leq s \leq K$ (Cook [6]), and

$$
\sigma_{s, k}=F(K, s, k) \quad(k \geq 4, s>K)
$$

(Baker [4]). For $k=2,3$ and $s>K$, see Baker [1,4]; for example, $\sigma_{3,2}=9 / 8$ and $\sigma_{5,3}=5 / 4$.

We refer the reader to Heath-Brown [8], Wooley [10], and Vaughan and Wooley [9] for results of the kind: for irrational $\alpha$, we have

$$
\left\|\alpha n^{k}\right\|<n^{-\tau_{k}}
$$

for infinitely many $k$. For example, one may take $\tau_{k}=1 / 9.028 k$ for every $k$ [10]. 


\section{BOUnds FOR WEYL SUMS}

We suppose throughout (as we may) that $\varepsilon$ is sufficiently small and $N$ is sufficiently large in terms of $k, \varepsilon$; we write $\eta=\varepsilon^{2}$.

Theorem 4. Let $k \geq 3$ and $\varepsilon>0$. Suppose that the Weyl sum

$$
g_{k}(\boldsymbol{\alpha} ; N):=\sum_{n=1}^{N} e\left(\alpha_{k} n^{k}+\cdots+\alpha_{1} n\right)
$$

satisfies

$$
\left|g_{k}(\boldsymbol{\alpha} ; N)\right| \geq A>N^{1-1 / 2 k(k-1)+\varepsilon} .
$$

Then there exist integers $q, a_{1}, \ldots, a_{k}$ such that

$$
1 \leq q \leq N^{\varepsilon}\left(N A^{-1}\right)^{k}
$$

and

$$
\left|q \alpha_{j}-a_{j}\right| \leq N^{-j+\varepsilon}\left(N A^{-1}\right)^{k} \quad(1 \leq j \leq k) .
$$

If $\alpha_{k-1}=\cdots=\alpha_{2}=0$, then the same conclusion holds with the weaker lower bound.

$$
\left|g_{k}(\boldsymbol{\alpha} ; N)\right| \geq A>N^{1-1 / k(k-1)+\varepsilon}
$$

in place of (2.1).

Proof. We initially proceed exactly as in the proof of [3, Theorem 4.3] with $\theta$ replaced by 0 and $\ell$ replaced by $(k-1) / 2$. This is permissible since we have

$$
J_{s, k-1}(N) \ll N^{s+\varepsilon}
$$

with $s=k(k-1) / 2$, in place of the bound for $J_{s, k-1}(N)$ used in [3]. We find that for $j=2, \ldots, k$ there are coprime pairs of integers $q_{j}, b_{j}$ with

$$
\begin{gathered}
1 \leq q_{j} \ll\left(N A^{-1}\right)^{k(k-1)}(\log N)^{C} \\
\left|q \alpha_{j}-b_{j}\right| \leq N^{-j+\varepsilon}\left(N A^{-1}\right)^{k(k-1)}
\end{gathered}
$$

where we shall use $C$ for an unspecified positive constant depending on $k$. Let $q_{0}$ be the l.c.m of $q_{2}, \ldots, q_{k}$. We now follow the argument of [3, pp. 41-42] to obtain

$$
q_{0} \ll(\log N)^{C}\left(N A^{-1}\right)^{k(k-1)} .
$$

It follows that, with $a_{j}=q_{0} b_{j} / q_{j}$, we have

$$
\left|q_{0} \alpha_{j}-\alpha_{j}\right| \leq N^{-j+2 \varepsilon}\left(N A^{-1}\right)^{2 k(k-1)} \quad(j=2, \ldots, k) .
$$

We now appeal to Lemma 4.6 of [3], which we restate here for clarity as Lemma 1 . 
Lemma 1. Suppose that there are integers $r, v_{2}, \ldots, v_{k}$ such that gcd $\left(r, v_{2}, \ldots, v_{k}\right)=1$,

$$
\left|q_{j} r-v_{j}\right| \leq N^{1-j} / 4 k^{4} \quad(j=2, \ldots, k),
$$

and that

$$
\left|g_{k}(\boldsymbol{\alpha} ; N)\right| \geq H>r^{1-1 / k} N^{\varepsilon} .
$$

There is a natural number $t \leq 2 k^{2}$ such that

$$
\begin{gathered}
t r \leq\left(N H^{-1}\right)^{k} N^{\varepsilon} \\
t\left|\alpha_{j} r-v_{j}\right| \leq\left(N H^{-1}\right)^{k} N^{-j+\varepsilon} \quad(j=2, \ldots, k) \\
\left\|\operatorname{tr} \alpha_{1}\right\| \leq\left(N H^{-1}\right) N^{-1+\varepsilon} .
\end{gathered}
$$

We now apply the lemma with $A=H, r=q_{0} d^{-1}, v_{j}=a_{j} d^{-1}$ where $d=\operatorname{gcd}\left(q_{0}, a_{2}, \ldots, a_{k}\right)$. From (2.5) and (2.6),

$$
\left|\alpha_{j} r-v_{j}\right| \leq N^{-j+2 \varepsilon}\left(N A^{-1}\right)^{2 k(k-1)} \leq N^{-j+1}\left(4 k^{4}\right)^{-1}
$$

since

and $r \leq N^{1-5 \varepsilon}$,

$$
\left(N A^{-1}\right)^{2 k(k-1)} \leq N^{1-12 \varepsilon}
$$

$$
A r^{-1+1 / k} N^{-2 \varepsilon} \geq N^{1-1 / k(k-1)-1+1 / k-C \varepsilon} \gg 1 .
$$

The inequalities (2.9)-(2.11) now yield the first assertion of the theorem with $q=t r$. For the second assertion, since $\alpha_{2}, \ldots, \alpha_{k-1}$ are 0 , we may take $r=q_{k}, v_{k}=b_{k}, v_{2}=\cdots=v_{k-1}=0, H=A$ in the application of Lemma 1. (The inequality (2.4) suffices in the earlier part of the argument.) We know that

$$
\left|r \alpha_{k}-a_{k}\right| \leq N^{-k+\varepsilon}\left(N A^{-1}\right)^{k(k-1)}
$$

rather than the weaker bound (2.6) . We may now complete the proof in the same way as before.

\section{Proof of Theorems 1, 2, and 3}

Proof of Theorem 11. Suppose there is no solution of

$$
1 \leq n \leq N,\left\|\alpha_{k} n^{k}+\cdots+\alpha_{1} n\right\| \leq N^{-1 / J+\varepsilon}
$$

where $J$ denotes $2 k(k-1)$. By [3, Theorem 2.2] we have

$$
\sum_{m=1}^{M}\left|g_{k}(m \boldsymbol{\alpha} ; N)\right|>N / 6,
$$

where $M=\left[N^{+1 / J-\varepsilon}\right]$. There is an integer $m, 1 \leq m \leq M$ such that

$$
\left|g_{k}(m \alpha ; N)\right|>A=N / 6 M \text {. }
$$


We have

$$
\left(N A^{-1}\right)^{2 k(k-1)} \ll M^{2 k(k-1)} \ll N^{1-2 k(k-1) \varepsilon} .
$$

By Theorem 4 there is a natural number $q=\operatorname{tr}$ such that

$$
\begin{aligned}
& q \ll N^{\varepsilon}\left(N A^{-1}\right)^{k} \ll M^{k}, \\
&\left\|q m \alpha_{j}\right\| \ll\left(N A^{-1}\right)^{k} N^{-j+\varepsilon} \\
& \ll M^{k} N^{-j+\varepsilon} \quad(j=1, \ldots, k) .
\end{aligned}
$$

Now let $n=q m$. Then

$$
\begin{aligned}
n \ll M^{k+1} & \ll N^{(k+1) / J} \ll N^{1-\varepsilon}, \\
\left\|n^{j} \alpha_{j}\right\| & \leq n^{j-1}\left\|n \alpha_{j}\right\| \\
& \ll M^{(k+1)(j-1)+k} N^{-j+\varepsilon} \ll M^{-1} N^{-\varepsilon}
\end{aligned}
$$

since $M^{(k+1) j} \ll N^{(k+1) j / J-(k+1) \varepsilon} \ll N^{j-2 \varepsilon}$. It follows that $n$ satisfies (3.1), which is a contradiction. This completes the proof of Theorem 1 .

Proof of Theorem (a). We follow the above proof; this time, $J$ denotes $k(k-1)$. The second assertion of Theorem 4 provides an integer $q=t r$ satisfying (3.2), and (3.3) for the relevant values $j=1, k$. Now we complete the proof as before.

Proof of Theorem 2(b). This is a simple consequence of Wooley's bound (1.2). Let $\nu=\nu(k)$ have the property that

$$
\min _{1 \leq n \leq N}\left\|\alpha n^{k}\right\| \ll_{k} N^{-\nu}
$$

for $N \geq 1$ and real $\alpha$. Let $a=\frac{1}{2+\nu}, b=1-a$. By Dirichlet's theorem there is a natural number $\ell \leq N^{b}$ with

$$
\left\|\alpha_{1} \ell\right\| \leq N^{-b}
$$

We now choose another natural number $m \leq N^{a}$ with

$$
\left\|\alpha_{k} \ell^{k} m^{k}\right\| \ll N^{-a \nu}=N^{-\nu /(2+\nu)} .
$$

Note that

$$
\left\|\alpha_{1} \ell m\right\| \leq N^{a-b}=N^{2 a-1} .
$$

Since $2 a-1=-\frac{\nu}{2+\nu}$, we have, with $n=\ell m$,

$$
1 \leq n \leq N,\left\|\alpha_{k} n^{k}+\alpha_{1} n\right\| \ll N^{-\nu /(2+\nu)} .
$$


Taking $\nu=1 / k(\log k+C \log \log k)$, we obtain

$$
\frac{\nu}{2+\nu}=\frac{1}{2 k \log k+2 C \log \log k+1},
$$

so that Theorem 2(b) holds with a suitable choice of $B$.

Example. If we take $k=20, \nu=1 / 222.16$ from [9], we obtain the value $1 / 445.32$ for $\rho_{20}$, which is not as good as Theorem 2(a). The proof of Theorem 2(b) is relatively crude, so it may be possible to do better using ideas from [9], [10].

Proof of Theorem [ (b). We can follow the proof of Theorem 1.8 of [4] (in the case $k \geq 4$ ) verbatim, replacing $K$ by $J:=k(k-1)$. The role of Lemma 5.2 of [4] is played by Theorem 4 in conjunction with [3. Lemma 8.6].

Proof of Theorem $3(a)$. Write $J=k(k-1)$ again. We assume that there is no solution of

$$
\left\|\beta_{1} n_{1}^{k}+\cdots+\beta_{s} n_{s}^{k}\right\| \leq N^{-s / J+\varepsilon}
$$

with $0 \leq n_{1}, \ldots, n_{s} \leq N,\left(n_{1}, \ldots, n_{s}\right) \neq \mathbf{0}$. Let

$$
S_{i}(m)=\sum_{n=1}^{N} e\left(m \beta_{i} n^{k}\right), \quad L=\left[N^{1 / J-\varepsilon}\right] .
$$

Following 44, Lemma 5.1, we find that there is a set $\mathcal{B}$ of natural numbers, $\mathcal{B} \subset[1, L]$, and there are positive numbers $B_{1} \geq \cdots \geq B_{s}$ such that

$$
B_{i}<\left|S_{i}(m)\right| \leq 2 B_{i} \quad(i=1, \ldots, s)
$$

and

$$
B_{1} \ldots B_{s}|\mathcal{B}| \gg N^{s-\eta} .
$$

(This may require a reordering of $\beta_{1}, \ldots, \beta_{s}$.) We can now follow the proof of Lemma 5.4 on [4], with $K$ replaced by $J$, to obtain the inequality

$$
|\mathcal{B}| \ll L N^{-1+2 k \eta}|\mathcal{B}|^{k / s} .
$$

Suppose first that $s>k$. Then

$$
L N^{-1+2 k \eta} \gg|\mathcal{B}|^{1-k / s} \gg 1,
$$

contrary to the definition of $L$.

Suppose now that $s \leq k$. Then

$$
\begin{aligned}
L^{\frac{k}{s}-1} & \geq|\mathcal{B}|^{\frac{k}{s}-1} \gg L^{-1} N^{1-2 k \eta}, \\
L & \gg N^{\frac{s}{k}-2 s \eta} .
\end{aligned}
$$


This is again contrary to the definition of $L$, and we conclude that there is a solution of (3.4).

\section{REFERENCES}

[1] R. C. Baker, Weyl sums and Diophantine approximation, J. London Math. Soc. (2) 25 (1982), 25-34. Correction, ibid. 46 (1992), 202-204.

[2] R. C. Baker, Small solutions of congruences, Mathematika 30 (1983), 164188.

[3] R. C. Baker, Diophantine Inequalities, London Mathematical Society Monographs, New Series, vol. 1, Oxford University Press, Oxford, 1986.

[4] R. C. Baker, Small solutions of congruences, II, Funct. et Approx. Comment. Math. 28 (2001), 19-34.

[5] J. Bourgain, C. Demeter, and L. Guth, Proof of the main conjecture in Vinogradov's mean value theorem for degrees higher than three, arXiv:1512.01565.

[6] R. J. Cook, The factional parts of an additive form, Proc. Camb. Phil. Soc. 72 (1972), 209-212.

[7] I. Danicic, Contributions to number theory, Ph.D. thesis, University of London, 1957.

[8] D. R. Heath-Brown, The fractional part of $\alpha n^{k}$, Mathematika 35 (1988), $28-37$.

[9] R. C. Vaughan and T. D. Wooley, Further improvements in Waring's problem, IV: Higher powers, Acta Arith. 94 (2000), 203-285.

[10] T. D. Wooley, The application of a new mean value theorem to fractional parts of polynomials, Acta Arith. 65 (1993), 163-179.

[11] T. D. Wooley, New estimates for smooth Weyl sums, J. London Math. Soc. (2) 51 (1995), 1-13.

[12] T. D. Wooley, Vinogradov's mean value theorem via efficient congruencing, II, Duke Math. J. 162 (2013), 673-730.

[13] T. D. Wooley, The cubic case of the main conjecture in Vinogradov's mean value theorem, arXiv:1401.3150.

[14] A. Zaharescu, Small values of $n^{2} \alpha(\bmod 1)$, Invent. Math. 121 (1995), 379388. 